\title{
A SUBFAMÍLIA GERRINAE (HEMIPTERA: HETEROPTERA: GERRIDAE) NA BACIA HIDROGRÁFICA DO RIO TROMBETAS, PARÁ, BRASIL.
}

\author{
Raquel T, de Moreira SAMPAIO', Victor PY-DANIEL ${ }^{1}$
}

RESUMO - Foi realizado um levantamento da Família Gerridae na Bacia Hidrográfica do rio Trombetas, Pará, Brasil, desde as cabeceiras até o Baixo Trombetas. Entre os Hemiptera aquático, Gerridae foi a Família mais representativa, sendo Gerrinae a subfamília mais coletada em 12 dos 35 pontos de coleta. Mapas com os primeiros registros da distribuiçao geográfica de Gerrinae para a Bacia do rio Trombetas e uma chave para a identificaçao dos cinco gêneros e seis espécies são apresentadas.

Palavras-chave: Insecta, Hemiptera, Gerridae, Insetos aquáticos.

The subfamily Gerrinae (Hemiptera: Heteroptera: Gerridae) at the Rio Trombetas hydrografic basin, Pará, Brasil.

ABSTRACT - A survey of the the Family Gerridae was made at the Hidrological Basin of the Trombetas river, Pará state, Brazil, since the Headwaters until the Lower Trombetas river. Gerridae was the most representative Family among the aquatic Heteroptera and Gerrinae the most collected subfamily on 12 of the 35 collected points. Maps with the first geographic distributional records for the Trombetas river Basin and a key for the identification of the 5 genera and 6 species is presented.

Key words: Insecta, Hemiptera, Gerridae, Aquatic Insects.

Os estudos sobre morfologia, discussões taxonômicas e distribuição geográfica de Gerridae na região Neotropical foram realizados em sua maioria por POISSON (1924), HUNGERFORD (1958a), HUNGERFORD e MATSUDA (1960), CALABRESE (1980) e ANDERSEN (1982). A distribuição dos hemípteros aquáticos foi abordada por JACZEWSKI (1937) e HUNGERFORD (1958b), restrita a aspectos de revisões descritivas. JACZEWSKI e KOSTROWICKI publicaram em 1969 alguns resultados sobre a fauna Holoártica destes grupos, discutindo afinidades entre a fauna e a região (ANDERSEN, 1982). Segundo CALABRESE (1980) as regiões Neártica e Paleártica não possuem gêneros endêmicos; 54 entre 57 gêneros existentes na Família Gerridae estão representados nos trópicos do Novo e Velho mundos, o que a leva a sugerir uma origem tropical para a Família. $\mathrm{O}$ levantamento dos gerrídeos da Bacia Hidrográfica do rio Trombetas foi realizado em convênio entre o Instituto Nacional de Pesquisas da Amazônia e a ELETRONORTE (Enge-Rio), no projeto de estudo de viabilização da construção da Hidrelétrica Cachoeira

1 Instituto Nacional de Pesquisas da Amazônia-INPA, Coordenação de Pesquisas em

Entomologia, Caixa Postal 478, 69011-970 - Manaus, Amazonas - Brasil 
Porteira, no rio Trombetas. Foram realizadas 35 coletas ao longo do rio Trombetas, Pará, desde o baixo Trombetas até a região dos formadores, incluindo o rio Mapuera até a cachoeira da Bateria. O material foi coletado com o auxílio de uma rede entomológica aquática ou rapiché nos remansos próximos às cachoeíras, trechos lênticos dos rios principais, às margens, em poças temporárias de águas de chuva ou cachoeira, igarapés de rio e à beira das estradas em Cachoeira Porteira, abrangendo todo o corpo de água que apresentasse condiçoes para o Gerridae se desenvolver. Foram encontrados gerrídeos em 12 dos 35 pontos de coleta na Bacia Hidrográfica do rio Trombetas. Todo o material coletado está depositado na Coleção de Invertebrados do Instituto Nacional de Pesquisas da Amazônia (INPA), Manaus, AM, Brasil.

\section{ÁREA DE ESTUDO}

A Bacia do rio Trombetas está compreendida entre as coordenadas geográficas $2^{\circ} 00^{\prime} \mathrm{S}-2^{\circ} 00 \mathrm{~N}$ e $55^{\circ} 30$ $59^{\circ} 00^{\prime} \mathrm{W}$ (fig 1 ), desde as cabeceiras do rio Trombetas na divisa do Brasil com a Guiana (rios Poana e Anamú), até a região do Baixo Trombetas (região do lago Erepecu), entrando pelo rio Mapuera até a Cachoeira da Bateria.

A sua macro-diferenciação com base tanto nos conjuntos geológicos como na própria dinâmica dos cursos d'água foi realizada da seguinte forma (fig 2) :

1) Baixo Trombetas, desde a confluência dos rios Mapuera e Trombetas até a foz onde não há formação de cachoeiras mas sim de "lagos" laterais às margens, onde o rio assume seu caráter Potamal, predominando a formação geológica sedimentar recente;

2) Zona de transiçãoTrombetas, do local denominado Cachoeira Porteira ao Remanso do Inferno ou Parana do Defunto, local onde o rio Trombetas abre-se formando uma rede de paranás ou canais. É onde ocorre uma interdigitação das áreas do précambriano e cenozóico;

\section{3) Zona de transição-Mapuera,} desde Cachoeira Porteira até a primeira grande cachoeira (Cachoeira da Égua);

4) Médio Trombetas, área compreendida entre o Remanso do Inferno e os formadores, excluindo-os, a qual está caracterizada por formaçoes précambrianas, águas lóticas, trecho apresentando diversas cachoeiras, típico trecho Ritral do rio;

5) Médio Mapuera, à partir da Cachoeira da Égua aos formadores (Tauini/Mapuerinha), analisado apenas até a Cachoeira da Bateria.

6) Formadores (cabeceiras), compreende apenas os formadores propriamente ditos do rio Trombetas: rios Poana e Anamu.

\section{Distribuição Geográfica da Subfamília Gerrinae na Região Amazônica.}

$\mathrm{Na}$ literatura existe em grande número registros quanto à distribuição genérica da Família Gerridae e em 


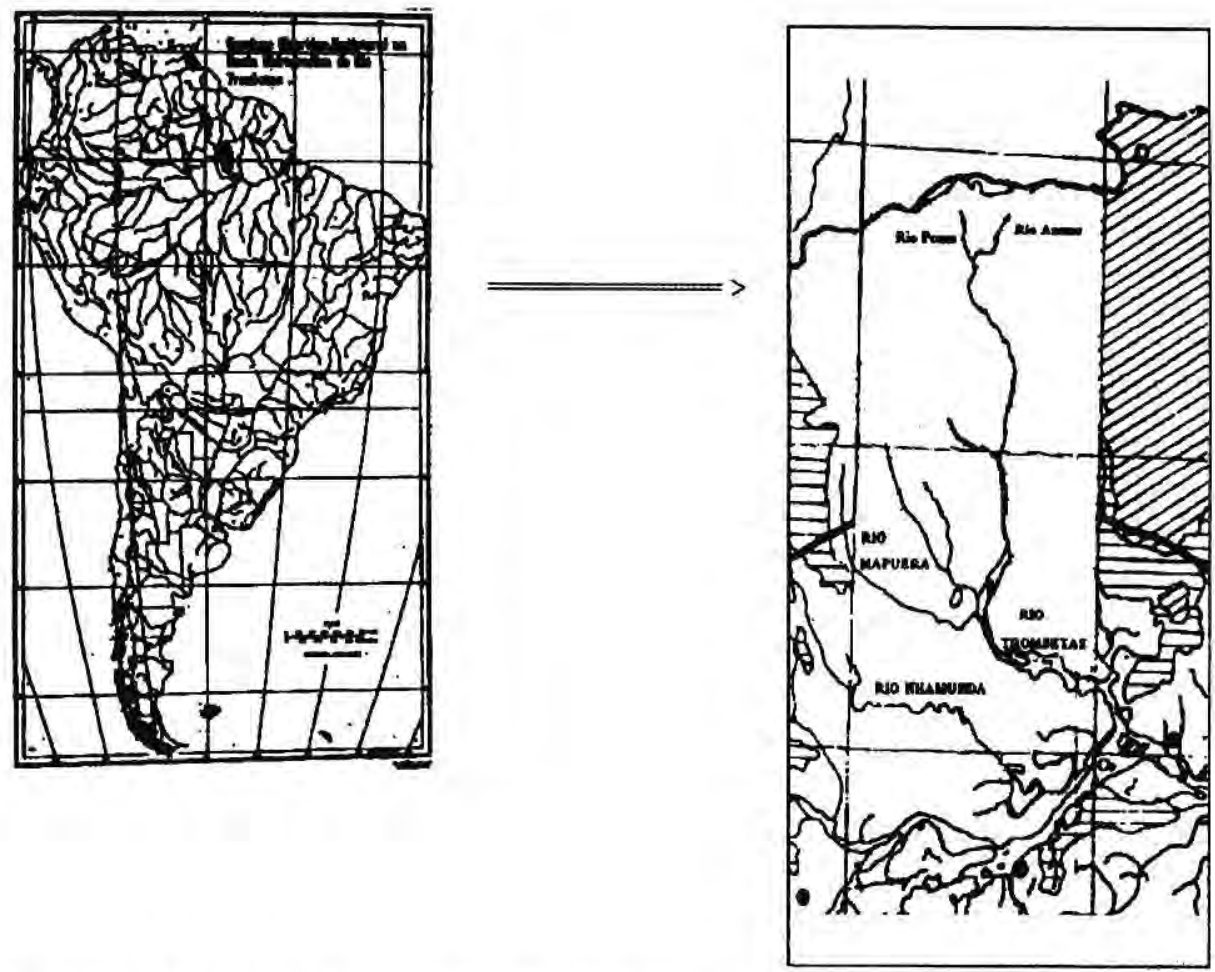

Figura 1. Mapa da América do Sul evidenciando a área trabalhada, Bacia Hidrográfica do Rio Trombetas, Pará, Brasil.

número reduzido para espécies. ela), Brachymetra (Bolívia, Brasil, HUNGERFORD \& MATSUDA, Guiana, Peru, Venezuela) e Eobates 1960, citam a distribuição para a (Peru). O gênero Limnogonus lt.s. é região amazônica dos seguintes gêneros: Tachygerris (Bolívia, Brasil, Peru e Suriname), Cylindrostethus (Brasil, Bolívia, Guiana, Peru), Potamobates (Colômbia, Peru, Venezuela), Charmatometra (Colômbia, Venezuconsiderado cosmopolita e Neogerris, subgênero de Limnogonus.

Dados organizados de distribuição geográfica das espécies descritas para a região amazônica, são inexistentes.

\section{Relaçao de Triboslgêneros\ espécies (sensu MATSUDA, 1960) na Bacia Hidrográfica do rio Trombetas.}

\section{Gerrini}

\section{Limnogonus Stal 1868}

recurvus Drake \& Harris, 1934

Neogerris Matsumura 1913 
celeris Drake \& Harris, 1934

lotus (White, 1878)

Tachygerris Drake 1957

surinamensis Hungerford \& Matsuda, 1958

Cylindrostethini

Cylindrostethus Fieber 1861

palmaris Drake \& Harris, 1934

Charmatometrini

Brachymetra Mayr1865

lata Shaw, 1933

Gênero Limnogonus Stal 1868

1868 - Limnogonus Stal, Herm. Fabr. 1:132.

1882 - Gerris (Lamprotrechus) Reuter, Oefv. Finska Vet. Soc. Forh. 25:40.

1898 - Limnogonus Champion, Biol. Centr. Amer., Rhynch. Heter. 2:151.

1909 - Limnogonus Kirkaldy e Torre-Bueno, Proc. ent. Soc. Wash. 1908, 10:210.

1911 - Gerris (Limnogonus) Torre-Bueno, Trans. Amer. ent. Soc. 32:244.

1917 - Tenagogonus van Duzee (nec Stal, 1855). Cat. Hem. : 429

1917 - Limnogonus Bergroth, Proc. Nac. Mus. 51 (2150):237.

1932 - Tenagogonus Anderson (nec Stal, 1855 ), Univ. Kansas Sc. Bull. 20:298.

1933 - Limnogonus Lundblad, Arch. Hydrob. Suppl. 12:374.

1934 - Limnogonus Drake \&
Harris, Ann. Carnegie Mus. 23:202205

1940 - Limnogonus Poisson, Bull. Soc. Sc. Bret. 17:(3-4):12-13.

1942 - Limnogonus Kuitert, Univ. Kansas Sc. Bull. 28:125

1958 - Limnogonus Hungerford, Proc. 10th. Intern. Congr. Ent. 1 : 346, f. 3.

1959 - Limnogonus Hungerford \& Matsuda, J. Kansas ent. Soc. 32(1): 40-41.

1960 - Limnogonus Hungerford \& Matsuda, Univ. Kansas Sc. Bull 41 (1):197-202.

Espécie assinaladas para a Bacia Amazônica : Limnogonus hyalinus (FABRICIUS, 1803); L. profugus Drake \& Harris, 1930; L. recurvus Drake \& Harris, 1930; L. ignotus Drake \& Harris, 1934; L. aduncus Drake \& Harris, 1938, var. uncatus Kuitert, 1942.

Tipo; Hydrometra hyalina (Fabricius) 1803 (por subseqüente designação de KIRKALDY, 1906) Localidade - tipo : "Americae Meridionalis aquis". 


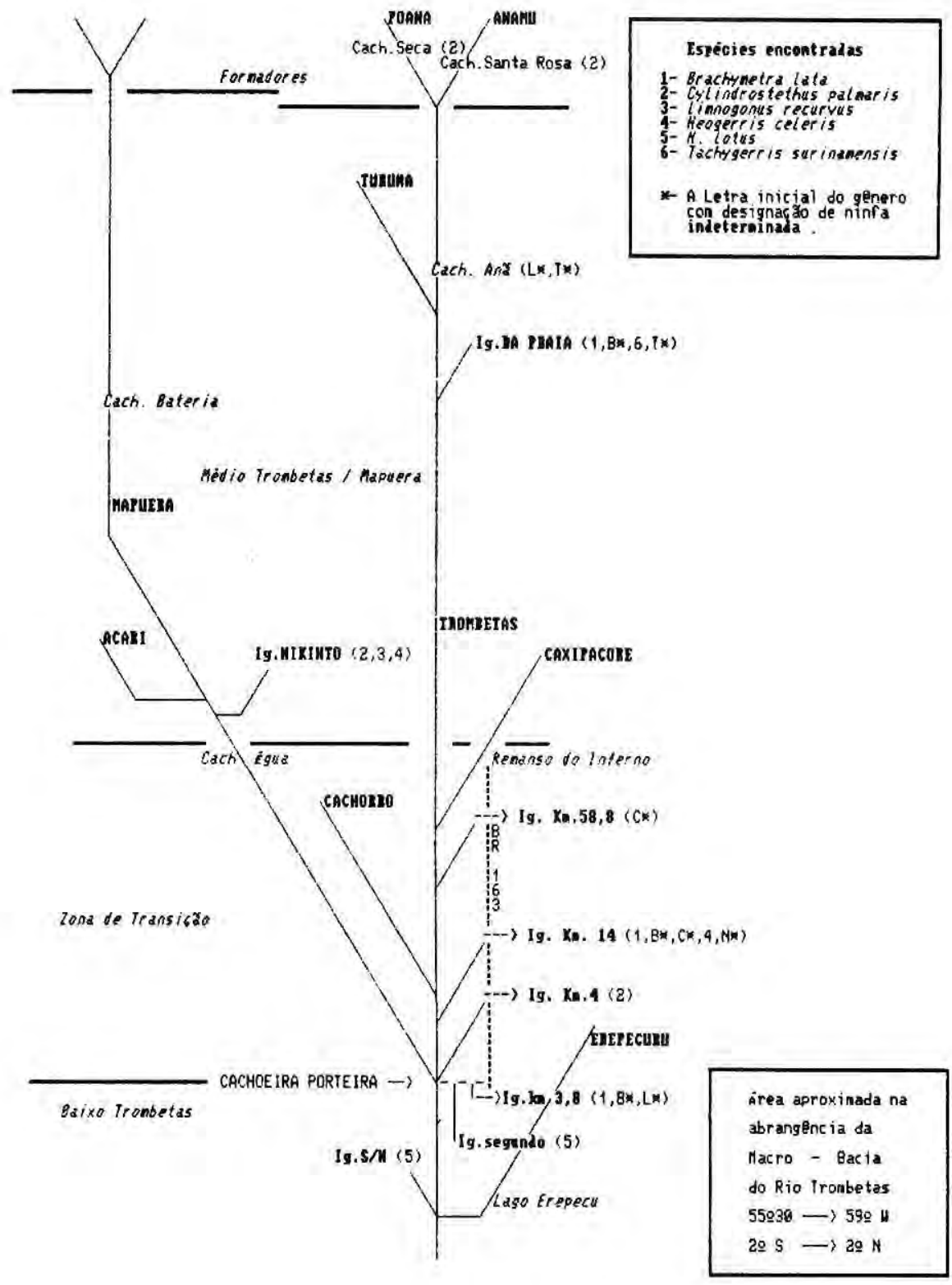

Figura 2. Distribuição Setorial da subfamília GERRINAE na Bacia Hidrográfica do Rio Trombetas. 
Limnogonus recurvus Drake \& Harris, 1934

Material coletado : BRASIL, Pará : Cachoeira Anã, rio Trombetas (Médio Trombetas), remansos em cachoeiras, 13.X.85, Equipe Comander (INPA), 02 fêmeas ápteras, 03 ninfas; Igarapé do $\mathrm{Km}$ 3,8 da Estrada que sai de Cachoeira Porteira para a Balsa, 01.X.85, Equipe Comander (INPA), 01 fêmea e 03 ninfas; Igarapé Nikihto, rio Mapuera, 04.VI.86, Equipe Baracuxí, 04 ninfas.

Observaçoes adicionais: $L$. recurvus foi coletada tanto em igarapés como na calha dos grandes rios, desde o Médio até o Baixo Trombetas, incluindo o rio Mapuera; a segunda espécie de maior dispersão.

L. recurvus foi encontrada em estômagos de Acarás (Characidae) e Traíras (Erythrinidae) pela pesquisadora R. GONÇALVES conforme informação do Dr. BENTO MASCARENHAS (MPEG).

\section{Neogerris Matsumura, 1913}

1913 - Neogerris Matsumura, Thous. Ins. Japan, Add. : 99.In ANDERSEN, N.M., 1982 Entomograph, vol.3. Scandinavian Science Press. Klapenborg-Denamark.

1930 - Limnogonus : ESAKI, Bull. Biogeogr. Soc. Japan 1 (3) : 214. in : HUNGERFORD, H. B. E., MATSUDA, R., 1961 - Insecta Matsumurana 24 (2) : 113.
1930 - Neogerris : MATSUMURA, Illustr. Thous. Ins. Japan $1 ; 14$. in ANDERSEN, N.M., 1982 - Entomograph, vol.3. Scandinavian Science Press.Klapenborg Denamak.

1935 - Limnogonus Lundblad, Arch. Hydrobil. Thous. Ins. Jap. 1(3): 214.

1959

Limnogonus

(Limnogonellus) HUNGERFORD \& MATSUDA, J. Kansas Sc. ent. Soc. 32:40-41.

1960 Limnogonus (Limnogonellus) HUNGERFORD \& MATSUDA, Univ. Kansas Sc. Bull. 41(1):11.

1960

Limnogonus

(Limnogonellus) MATSUDA, Univ. Kansas Sc. Bull. 41(1):200.

1961 - Limnogonus (Neogerris) HUNGERFORD \& MATSUDA, Insecta Matsumurana 24:112-114.

1963 - Limnogonus (Limnogonellus) POISSON, Bull. Inst. fr. Afr. noire (A)25:1178.

1965 - Neogerris POISSON, Bull. Inst. fr. Afr, noire (A)27:1488.

Espécies assinaladas para a Bacia Amazônica: Neogerris lotus (WHITE, 1878); N. lubricus (WHITE, 1879); N. celeris Drake \& Harris, $1934 ; N$. celeris var. magnus Kuitert, $1942 ; N$. genticus Drake \& Harris, 1934; N. visendus Drake \& Harris, 1934.

Espécie-tipo: Neogerris boninensis Matsumura 1913 (monobásico). 
Localidade-tipo: Ogazawarajima, Bonin Is., Japão.

\section{Neogerris celeris Drake \&}

\section{Harris, 1934}

Material coletado: Brasil, Pará: Igarapé do Km 14, Estrada BR 163, Cachoeira Porteira (Zona de Transição), 14-15.VI.88, Equipe Pyriquito, 01 fêmea; Igarapé Nikihto, rio Mapuera, 04.VI.86, Equipe Baracuxí, 05 ninfas; Lago Zibeira, rio Trombetas (Baixo Trombetas), 13.III.86, Equipe Apoidea (INPA).

Observaçoes adicionais: Esta espécie foi coletada apenas em igarapés, no rio Mapuera, Zona de TransiçãoTrombetas e Baixo Trombetas.

\section{Neogerris lotus (White, 1879)}

Material coletado: BRASIL, Pará: Igarapé SN na margem direita do rio Trombetas (Baixo Trombetas), 08.III.86, Equipe Apoidea (INPA), 01 fêmea; Igarapé $\mathrm{S} \backslash \mathrm{N}$, rio Mapuera, 04.VI.86, Equipe Baracuxí (INPA), 02 fêmeas.

Observações adicionais: $N$. lotus foi apenas coletada em igarapés e no Baixo Trombetas.

Gênero Tachygerris Drake, 1957

1853 - Tenagogonus STAL, Ofv. vet. Akad. Forh 10:263. $12: 45$

1855 - Tenagogonus STAL, ibid.

1865 - Limnometra MAYR,
Verz. Zool. - bot. Ges. Wien. 15:443. 1898 - Limnometra CHAMPION, Biol. Cent. Amer., Rhynch. 2:150-151

1909

Tenagogonus

KIRKALDY e TORRE-BUENO, Proc. ent. Soc. Wash. 10:209

1915

Tenagogonus

BERGROTH, Zoolgische Medeelingen uitgegeven vanwegels Rijks Museum van Natuurlijke Historiete Leiden, 1 (2) : 121-123.

1925 - Limnometra CHINA Bull. Brooklyin ent. Soc. $20: 218$. in : HUNGERFORD, H. B. \& MATSUDA, R., 1958 - Univ. Kansas Sc. Bull 39(9): 375.

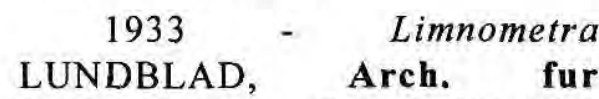
Hydrobiologie, Suppl. Bd 12:1 "Tropische Binnengewasser" 4:388392.

1934 - Tenagogonus DRAKE \& HARRIS, Ann. Carn. Mus. 23:131134

1942 - Tenagogonus KUITERT, Un. Kansas Sc. Bull. 28:131-135.

1957 - Tachygonus DRAKE,

Proc. Biol. Soc. Wash. 70:111.

1957 - Tachygerris DRAKE, Proc. Biol. Soc. Wash. 70:193.

1958

Tachygerris HUNGERFORD \& MATSUDA, Jour. Kansas ent. soc. 31 920:113115.

1960 - Tachygerris HUNGERFORD \& MATSUDA, Univ. Kansas Sc. Bull. 41 (2):10.

1960 - Tachygerris MATSUDA, 
Univ. Kansas Sc. Bull. 41(2):202-205.

Espécies assinaladas para a Bacia Amazônica: Tachygerris celosis (Drake \& Harris, 1931), T. spinulatus (Kuitert, 1942), T. opacus (Champion, 1901), $T$. adamsoni (Drake, 1940) E $T$. surinamensis (Hungerford \& Matsuda, 1958).

Espécie-tipo : Tachygerris adamsoni Drake, 1940.

Tenagogonus adamsoni Drake, 1940 (por descrição original)

Localidade-tipo : Bolívia

Tachygerris surinamensis Hungerford \& Matsuda, 1958

Material coletado: BRASIL, Pará: Igarapé da Praia, Estirão da Fumaça, rio Trombetas (Médio Trombetas), 14.X.85, Equipe Comander, 01 fềmea, 01 macho, 02 ninfas; Cachoeira Anã, rio Trombetas, 13.X.85, Equipe Comander, 01 ninfa.

Observações adicionais: $T$. surinamensis foi coletada tanto em igarapés como na calha dos grandes rios e apenas foi encontrada na região do Médio Trombetas, sendo a espécie de menor dispersão na Bacia do rio Trombetas.

\section{Cylindrostethus Fieber, 1861}

1860 - Cylindrostethus Fieber, Europ. Hemip.: 33. in: MATSUDA, R., 1960 - Univ. Kansas Sc. Bull., 41(2):224.

1865 - Cylindrostethus MAYR,
Verh. Zool. Bot. Ges. Wien, 15:444

1897 - Cylindrostethus KIRKALDY, Entomologist, 30:258

1902 - Cylindrostethus

BERGROTH, Ent. Month. Mag. 18:258.

1908 - Cylindrostethus KIRKALDY \& TORRE-BUENO, Proc. ent. Soc. Wash., 10:210.

1915 - Cylindrostethus SCHMIDT, Stett. Ent. Zeit., 76:361. 1925 - Cylindrostethus TORREBUENO, Spolia Zeylandica, 13:226.

1934 - Cylindrostethus DRAKE \& HARRIS, Ann. Carnegie Mus., 23:179-240.

1933 - Cylindrostethus LUNDBLAD, Arch. Hydrobiol. Suppl. 12, Tropische Biennengewasser, 4:392-394.

1942 - Cylindrostethus KUITERT, Univ, Kansas Sci. Bull., 28:135-138.

Espécies assinaladas para a Bacia Amazônica: Cylindrostethus bassleri Drake, 1952, C. bilobatus Kuitert, 1942 , C. erythropus (Herrich-Schaeffer, 1850), C. hungerfordi Drake \& Harris, 1934, C. linearis Erichson, 1848, C. palmaris Drake \& Harris, 1934.

Espécie-tipo: C. fieber Mayr, 1865 (= G. productus (Spinola, 1840)). Localidade-tipo: Ceilão (= Sri-Lanka).

\section{Cykindrostethus palmaris Drake \& Harris, 1934}

Material coletado: Brasil, Pará: Cachoeira Santa Rosa, rio Anamu 
(Formador do rio Trombetas), 11.X.85, Equipe Comander (INPA), 02 machos, 04 fêmeas; Cachoeira Seca, rio Poana (Formador do rio Trombetas), 10.X.85, Equipe Comander (INPA); Igarapé do $\mathrm{Km} 58,8$, Estrada BR 163, Cachoeira Porteira (Zona de Transição), 16.IV.85, Equipe Pyriquito (INPA), 02 ninfas; Igarapé do $\mathrm{Km} \mathrm{14,} \mathrm{Estrada} \mathrm{BR} \mathrm{163,}$ Cachoeira Porteira, 15 e 17.IV.85, Equipe Pyriquito (INPA), 02 ninfas, 08 fêmeas, 04 machos; Igarapé Nikihto, rio Mapuera, 04.VI.86, Equipe Baracuxí (INPA), 02 ninfas; Igarapé do Cordeiro, rio Mapuera, 27.IV.85, Equipe Pyriquito (INPA), 01 ninfa; Igarapé do Borges, rio Mapuera, 27.IV.88, Equipe Pyriquito (INPA), 01 fêmea.

Observações adicionais: Esta espécie foi encontrada tanto em igarapés como na calha dos grandes rios, sendo a que apresentou maior dispersão ao longo da Bacia Hidrográfica do rio Trombetas. Registra-se ainda a predação de $C$. palmaris por quelônios (Podocnemis expansa) na Reserva Biológica Guaporé em Rondônia.

\section{Brachymetra Mayr, 1865.}

1865 - Brachymetra MAYR, Verh. Zool. - bot. Ges. Wien, 15:445.

1933 - Brachymetra SHAW, Univ. Kansas Sc. Bull., 21:221-222.

1945 - Brachymetra HARRIS \& DRAKE, Proc ent. Soc. Wash., 47(7): 212.

1960 - Brachymetra HUNGERFORD \& MATSUDA, Univ. Kansas Sc. Bull., 41(1):9.
1960 Brachymetra MATSUDA, Univ. Kansas Sc. Bull., 41(2):240-242.

Espécies assinaladas para a Bacia Amazônica: Brachymetra lata Shaw, 1934, B. furva Drake, 1957, B. mera Harris \& Drake, 1945, B. albinerva incisa Shaw, 1934, B. shawi Hungerford \& Matsuda, 1957, B. kleopatra Kirkaldy, 1899, B. anduzeei Drake \& Harris, 1942, B. accola Drake, 1957.

Espécie-tipo: $B$. albinerva (AMYOT \& SERVILLE, 1843).

Localidade-tipo: América do Sul (Trinidad).

Brachymetra lata Shaw, 1933

Material coletado: BRASIL, Pará: Igarapé da Praia, estirão da Fumaça, 14.X.85, Equipe Comander (INPA), 03 machos, 03 fêmeas, 05 ninfas; Igarapé do Km 14, Estrada BR 163, Cachoeira Porteira, 15.IV.85, Equipe Pyriquito, 06 ninfas, 03 fềmeas,

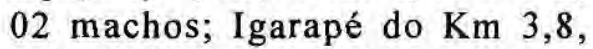
Estrada de Cachoeira Porteira para a Balsa (Zona de Transição), 01.X.85, Equipe Comander, 03 fêmeas.

Observações adicionais: Brachymetra lata foi apenas coletada em igarapés, desde o Médio à Zona de Transição-Trombetas.

\section{Comentários Gerais}

Com base na análise das espécies coletadas é possível sugerir que a diversidade faunística na Bacia do rio Trombetas, dentro de 
Gerrinae, apresenta-se maior nas regiões de contacto entre áreas sedimentar/précambriana, e que tende a diminuir tanto em direção à fóz como para os formadores (cabeceiras).
A Família Gerridae, entre todos os Hemiptera aquático coletados na Bacia Hidrográfica do rio Trombetas, apresentou-se como a mais representativa, $40,2 \%$ ( 890 exemplares em um total de 2213).

\section{Chave para as espécies de Gerridae encontradas na Bacia Hidrográfica do rio Trombetas, Pará.}

1. Tubérculos anteníferos direcionados lateralmente; tórax cilíndrico, não dilatado ântero-ventral (vista lateral); pronoto não expandido em lobo posterior; quarto segmento do rostro não ultrapassa a coxa anterior; metanoto $=$ metade do mesonoto, mesonoto com listras conspícuas em cada lado; primeiro segmento genital do macho terminando em ponta fina, lobo superior do segundo segmento expandido lateralmente em estruturas semelhantes às abas de um prato Cylindrostethus palmaris.

- Tubérculos anteníferos direcionados ântero-lateralmente; tórax dilatado antero-ventral (vista lateral); pronoto com lobo posterior expandido; quarto segmento do rostro ultrapassa a coxa anterior em pelo menos seu comprimento, mesonoto, metanoto e genitália do macho com outro aspecto. 2.

2. Antena mais longa que o corpo; segundo segmento antenal muito menor que o terceiro, quase a metade; primeiro segmento antenal menor que o terceiro; terceiro segmento do rostro maior que oito vezes o comprimento do segundo Tachygerris surinamensis.

- Antena mais curta que o corpo, raramente tão longa quanto o corpo; segundo segmento antenal maior que a metade do terceiro; primeiro segmento antenal maior ou igual ao terceiro; terceiro segmento do rostro igual ou menor que oito vezes o comprimento do segundo 3.

3. Distância tubérculo antenífero à fronte (vista dorsal), subigual à largura dos olhos; comprimento dos olhos subigual ou pelo menos menor que duas vezes a largura dos olhos 4.

- Distância tubérculo antenífero fronte (vista dorsal) maior ou menor que a largura dos olhos; comprimento dos olhos pelo menos duas vezes a largura. .... 5 .

4. Fêmur anterior sem setas na região basilar posterior próxima ao trocânter; tórax opaco; pronoto com duas listras paralelas medianas no lobo anterior; distância interocular pelo menos três vezes e meia maior que a distância tubérculo antenífero à fronte (vista dorsal), ápice do conexivo com projeções semelhantes a espinhos terminais Limnogonus recurvus. 
- Fêmur anterior com pelo menos quatro setas curtas na região basilar posterior próxima ao trocânter; tórax brilhante; pronoto com mancha clara oval no lobo anterior; distância interocular é apenas metade maior que a distância tubérculo antenífero à fronte (vista dorsal); ápice do conexivo sem espinhos terminais Neogerris celeris.

5. Margem posterior dos olhos ultrapassam o ângulo ântero-lateral do pronoto; tricobótrias cefálicas marcadas por manchas marrom avermelhadas; setas oculares conspícuas; fronte arredondada; abertura da glândula odorífera conspícua; presença de sutura intersegmental secundária; fêmur anterior com pelo menos quatro setas longas na região basilar posterior ao trocanter e uma fileira de espinhos curtos na margem interna desde a base até o ápice ....... Brachymetra lata.

- Olhos globosos; a margem posterior não ultrapassa o ângulo ânterolateral do pronoto; cabeça escura dorsalmente, manchas marcando tricobótrias não conspícuas; setas oculares não conspícuas; ápice da cabeça projetado anteriormente; abertura da glândula odorífera não conspícua; sem sutura intersegmental secundária; fêmur anterior com cerca de onze setas curtas na região basilar posterior e sem espinhos curtos na margem interna; pubescência branca brilhante na mesa e metacetábulas e sutura metacetabular dorsalmente ....... Neogerris lotus.

\section{Referências bibliográficas}

ANDERSEN, N. M. 1982. The Semiaquatic Bugs ( Hemiptera, Gerromorpha). Phylogeny, Adaptations, Biogeography and Classification. Entomograph, vol 3. Scandinavian Science Press. Klapenborg - Denmark, $455 \mathrm{p}$.

ANDERSON, L. D. 1932. A monograph of the genus Metrobates Hemiptera, Gerridae). Univ. Kansas Sc. Bull., 20(16):298.

BERGROTH, E. 1902. On the Thorax of the Gerridae. Entomologist mon Mag, (2) 13(38):258-260.

BERGROTH, E. 1915. Some Javanese Hemiptera Collected by E. Jacobson and Th. H. Mac Gillavry. Zool Meded., 1:109123.

BERGROTH, E. 1916. New and Little Known heteropterous Hemiptera in the United States National Museum. Proc. U.S. natn. Mus., 51: 215-239.

CALABRESE, D. M. 1980. The zoogeography and Cladistic Analysis of the Gerridae
(Hemiptera: Heteroptera ). Misc. Publ. ent. Soc. Am., 11:1-119.

CHAMPION, G. L. 1898. LimnometraLimnogonus. Rhynchota, Heteroptera. Biologia cent. - am., (2):150-151.

DRAKE, C. J. 1957. A necessary correction in the nomenclature of the water-striders. Proc. biol. Soc. Wash., 70:193-194.

DRAKE, C. J.; HARRIS, H. M. 1934. The Gerrinae of the Western Hemisphere (hemiptera). Ann. Cameg. Mus., 23:179. 240.

HARRIS, H. M.; DRAKE, C. J. 1945. A new Brachymetra from Peru with a list of known species (Hemiptera, Gerridae). Proc. ent. Soc. Wash, 47(7):211-212.

HUNGERFORD, H. B. 1958a. Some interesting aspects of the world distribution and classification of Aquatic and Semiaquatic Hemiptera. Proc. X. Congr. Ent. Montreal 1956, 1:337-348.

HUNGERFORD, H. B. 1958b. Two new genera of Gerridae with the description of a new species. J. Kans. ent. soc., 
31(2):113-117.

HUNGERFORD, H. B.; MATSUDA, R. 1958.

The Tenagogonus-Limnometra Complex of the Gerridae. Univ. Kansas. Sc, Bull., 39(9):375.

HUNGERFORD, H. B.; MATSUDA, R. 1959. Concerning the genus Limnogonus and a new subgenus (Heteroptera: Gerridae). $J$. Kansas ent. Soc., 32(1):40-41.

HUNGERFORD, H.B.; MATSUDA, R. 1960. Keys to subfamilies, tribes, genera and subgenera of the Gerridae of the World. Kansas U. S. B., 41:3-23.

HUNGERFORD, H. B.; MATSUDA, R. 1961. A review of the subgenus Neogerris Matsumurana (Hemiptera: Gerridae). Insecta matsumu., 24 (2):112-114.

JACZEWSKI, T. 1937. All gemeine Zuge der geographischen Verbreitung der Wasserhemipteren.Arch, Hydrobiol, 31:565-591.

JACZEWSKI, T.; KOSTROWICKI, A. S. 1969. Number of species of aquatic and semiaquatic Heteroptera in the fauna of various parts of Holartic in relation to the World fauna. Memoire Soc. ent. ital., 48:153-156

KIRKALDY, G. W. 1897. Synonymic notes on aquatic Rhynchota. Entomologist, 30:258-260.

KIRKALDY, F. E. S. G. W.; BUENO, J.R. de La T. 1909. A Catalogue of American Aquatic and Semi-aquatic Hemiptera. Proc. ent. Soc. Wash., 10:173-215.

KUITERT, L. 1942. Gerrinae in the University of Kansas Collection. Kansas U. S. B., 28:125-131.

LUNDBLAND, O. 1993. Zur Kennitnis der Aquatilen und semiaquatilen Hemipteren von Sumatra, Java und Bali. Arch. Hydrob. Suppl., 2, Tropische Biennengewasser, 4:1-195, 263 - 489.

MATSUDA, R. 1960. Morphology, Evolution and a classification of the Gerridae (Hemiptera:Heteroptera). Kansas $U . S$. B., 41:21-632.

MAYR, G. L. 1865. Dagnosen neuer Hemipteren 2. Verh. Zoolbot. Ges. Wien, 15:429-446.

POISSON, R. 1924. Contributions a l'etude des Hemipters aquatiques. Bull. Biol. France Belg., 58:49-305.

POISSON, R. 1940. Contribution á l'etude des Hydrocorises de Madagascar (4e Mémoire). Mem. Inst. scient. Madagascar (E), 7:243 -265.

POISSON, R. 1963. Mission de M. H. Berthand (1958 - 1960) en Afrique éthipienne et a Madagascar. Hydrocorises. Bull. Inst. fr. Afr. noire (a), 25:1170-1207.

POISSON, R. 1965. Catalogue des insectes Héteroptères Gerridae Leach, 1807, africano - malgaches. Bull. Inst. fr. Afr. noire, 27: 1466 - 1503.

REUTER, O. Om. 1883. Ad cognitionem Heteropterorum Africae Occidentalis. Oefv. Finska. Vet. Soc. Forh., 25:40.

SHAW, G. J. 1933. A study of the genus Brachymetra (Hemiptera : Gerridae). Kansas U. S. B., 21:221-233.

SCHMIDT, 1915. Zur Kennth. d. Gen. Saceseurus Br. u. Cylindrostethus Fieb. Stettiner Ent. Zeitung, 76:361-364.

TORRE-BUENO, J. R. 1911. The Gerrids of the Atlantic states. Trans. Am. ent. Soc., 37(3):243-252.

TORRE-BUENO, J. R. De La. 1952. On some Aquatic Hemiptera from Ceylon with descriptions of new forms. Spolia zeylan., 13:223-234,

VAN DUZEE, E. P. 1917. Catalogue of the Hemiptera of America north of Mexico, excepting the Aphidae, Coccidae and Aleurodidae. - Univ. Calif. Publ. Tech. Entomol., 2:1-902. 